\title{
The One-Dimensionality of Scientific Relativism
}

\author{
János Laki \\ Institute of Philosophy, Research Centre for the Humanities, Hungarian Academy of Sciences, Hungary/ \\ Laki.Janos@btk.mta.hu
}

\begin{abstract}
The historicist approach to science has been accompanied by a culturalist one in the last decade or two. Epistemic localism added a horizontal axis to the existing vertical (historical) one thus science came to be presented in a coordinate system as a manifold of epistemic traditions. Taking the debate about the existence of the N-ray as an instructive example, I argue that the historical development of science creates disciplinary communities that impose unified epistemic standards on the communities scrutinizing the same aspects of reality. Accordingly, with the advent of such communities relativism became one-dimensional: science has developed into a historically changing culture that puts up a successful fight against epistemic diversity in its synchronous dimension.
\end{abstract}

Keywords: epistemic systems, geographical turn, local epistemologies, N-ray, objectivity, relativism

\section{Introduction: Two kinds of cultural relativism}

The historical approach to science in the middle of the last century revealed that scientists in different eras use incompatible concepts, methodological norms and epistemological standards for constructing and justifying scientific knowledge. Sociologists and geographers of the last decades went on with this destruction of the unity of science claiming that scientific knowledge "... is not to be thought of as some transcendent entity that bears no trace of the parochial or contingent. It needs, rather, to be qualified by temporal and regional adjectives" (Livingstone, 2003: 13, my italics). Thereby a "geographical" or "spatial turn" has been added to the historical one, creating a new dimension of relativism in science. The protagonists of this spatialist approach agree that the “... issues of space - location, place, site, migration, region - are at the heart of scientific endeavour" (Livingstone, 2003: 5, my emphases) and with "the 'geographical turn' evident across science studies ... different geographies of science are emerging" (Powell, 2007: 309). The addition of this further "turn" to the already existing ones is meant to indicate that concepts and standards of science vary with regions, therefore "just as there is a rich history of science, so there is a rich geography of science" (Withers and Livingstone, 2011: 3). It is claimed that the "... processes of knowledge production" require "judgements and negotiations by groups of scientists in specific contexts". Accordingly, cartographers of science take on the task to reveal "the specific sites" at which "... particular scientists with particular skills, materials, tools, theo- 
ries and techniques" (Turnbull, 2002: 6, my italics) produce locally authenticated beliefs.

The same cultural relativism can be found in contemporary sociology of scientific knowledge as well. From the fact that this knowledge is "fabricated and negotiated by particular agents at a particular time and place", some sociologists come to the conclusion that it is "local rather than universally valid" (Knorr-Cetina, 1981: 33, my italics). Their conclusion is based on the premise that human reason and empirical evidence in themselves are epistemically not powerful enough, so they conceive knowledge in general as an "amalgam of experiences and socially mediated beliefs" (Bloor, 1976: 12). The social mediation of beliefs is concretised as setting up justificatory systems authorised by particular communities, therefore they look for "the specific local causes of credibility" (Barnes and Bloor 1982: 23 - my italics). Which beliefs are given credit depends on the epistemic standards a community deploys thus belief and knowledge differ not in the latter's being justified (and true), but in its being "collectively endorsed" (Bloor, 1976: 2-3), hence epistemic justification is to be replaced by acceptance (Bloor, 1976: 2-3).

\section{The anthropological treatment of local relativism}

Naturally, "spatiality" (just like "temporality") itself cannot be more than just a synecdoche: spatial or temporal coordinates have nothing to do with the justification of beliefs. What does have such an impact is the epistemic system people use for assessing statements. It is an "assemblage of principles and procedures that a given society or culture ... relies on, explicitly or implicitly, in distinguishing justified from unjustified beliefs..." (Williams, 2007: 94). It is often said that "e]pistemic systems vary from culture to culture..." (Williams, 2007: 94), but this does not involve that "culture" is synonymous with "being separated by a physical distance". There are different cultures in the same geographical region and the other way round, people unified by the same (sub)-culture may not be living in the same place. People of the same scientific school, tradition, paradigm etc. can adhere to the same commitments independent of their temporal or geographical location. Platonism, for instance, can be taken as an epistemic system accepted by people not belonging to the same historical era or geographical area. Certainly, cultural relativism used to be coupled with spatiality while communication, hence the authorisation of particular standards, presupposed direct, unmediated communication and interactions. Thus in the course of history epistemic systems differed due to their physical separateness, but geographical distance is not a necessary condition of such differences. What is necessary is a community that shares customs, traditions and authority structure that empowers the standards and adjudicates whether in concrete cases they are observed.

Such communities may materialize in contemporary science without their members being separated by physical distance (Longino, 2002; Coliva and Pedersen, 2017). However, the cultural relativism such communities generate is not easy to reconcile with the cognitive success of science hence attempts have been made to explain the cooperation among such traditions. One of the most ingenious of them was put forward by $P$. Galison who argued that the prima facie fragmentariness of science is a consequence of identifying holistic unities that make "unbridgeably isolated" knowledge blocks, "island empires" (Galison, 1997a: 17-18) using "incommensurable languages ... without a common divisor" (Galison, 2010: 42). He suggests that this vision is induced by the assumption that science must be a compact culture integrating theory, experimentation and instrumentation. Scientists themselves perceive their situation differently, therefore they do not worry about relativism. They can put up with the fact that science falls into more or less discrete subcultures, because they experience that, despite the incommensurable languages they use, their communities can escape "the methodological and philosophical commitment to relativism" (Galison, 2010: 42). The relatively independent, but partially overlapping communities, no matter where their members are geographically located, share concepts and practices enough to allow rational communication. Taking neighbouring lay cultures as his model, Galison describes the interfaces between separate scientific communities as "trading zones" where collaboration and repeated attempts at communication create interlanguages by a hybridisation of the particular idioms. Admittedly, such inter-languages provide 
only partial competence, but Galison claims that it is enough for mutual understanding and eschewing "block relativism" (Galison, 1997a: 14).

A slightly different still similar approach was suggested by Collins and Evans who distinguished between "contributory" and "interactive expertise", claiming that while the former presupposes full participation in the research process, the latter can be acquired by joining in discussions and collaboration with the competent speakers of the community to be understood (Collins and Evans, 2015 , 2016). The interactive expertise obtained this way is restricted again, but enough for partial understanding and it allows discussion that can result in bridging over the conceptual gaps.

Galison, Collins and Evans alike use the cultural anthropological model of mediation between alien cultures for soothing the severe consequences of localist relativism in science. The shared aim of the conceptual innovations of "trading zone" and "interactive expertise" is to explain how mutual (though partial) understanding can be created by local interactions without homogenising the global conceptual schemes of the parties. Seeing the similarity of their pursuits, M. E. Gorman (Gorman, 2002), then Collins, Evans and Gorman together (Collins et al., 2007; cf. Collins et al., 2017) came up with the idea that by the combination of Galison's and Collins and Evans' ideas we can gain a framework suitable for dealing with incommensurable traditions and making thereby the inter- and multidisciplinary research possible (in what follows I refer to this as 'G-C-E framework').

I would argue that the anthropological method the G-C-E framework suggests may be suitable for handling the semantic problems caused by incongruent conceptual apparatuses, but it leaves the door open for epistemic relativism. The framework makes use of the linguistic and interactive relations of the communities: shared observable situations and interactions can help accommodate incongruent concepts and interpretations. Epistemic relativism, however, is caused by the incongruity of the deep-rooted standards by which evidence are gained from the observable situations and the epistemic merits of assertions are determined. Since these are culturally ingrained commitments, understanding of them is not identical with accepting their validity, hence the G-C-E framework alone is not sufficient for overcoming cultural relativism.

In what follows, I argue that science is a special culture that includes an urge to homogenise the epistemic system used. I present my position by means of a case study revealing how contradictory statements concerning a new kind of electromagnetic radiation were treated at the beginning of the $20^{\text {th }}$ century. The case itself is known (see Blondlot, 1905a; Klotz, 1980, 1986; Langmuir, 1989; Nye, 1980, 1986; Seabrook, 1941), hence I am not going to rehearse all its details. I only highlight the features that the standard history leaves obscure though they shed light on the mechanism of epistemic homogenisation.

\section{The rise of a local tradition}

Here is the bald summary of the official story: In spring 1903, R. Blondlot announced the discovery of a new radiation that he named $\mathrm{N}$-ray. After several failed attempts to replicate his experiments by other physicists, R. W. Wood visited Blondlot's laboratory and conducted there an experimentum crucis that empirically refuted this claim. "Wood rather cruelly published what happened in the laboratory. And that was the end of Blondlot" (Langmuir, 1989: 43).

The received explanation of the issue is that, tricked by his strong expectations, an individual scientist imagined seeing signs (namely brightness-changes of sparks) that in fact were not there. Thus he fell prey to self-delusion. As a matter of fact, however, the N-ray story was much more complicated than its standard rendering makes us believe. It is worth taking a look at the details. ${ }^{1}$

1. The existence of the N-ray fitted very well in the knowledge context of the era:

More than one kind of radiation (X-ray, alpha-, beta- and gamma-ray, natural radioactivity) had been discovered in the preceding years, hence a further one could be expected.

2. Blondlot's and others' results went through the official filters of science:

A huge number of papers were published about the topic in peer reviewed scientific journals (120 researchers published about 300 papers in a short period of time). 
Lectures were delivered about the N-ray at the French Academy of Sciences and in prestigious learned societies.

Indeed, the significance of the discovery became so obvious that even priority debates broke out among scientists (Le Bon, Audollet, Huter vs. Charpentier).

3. The hypothesis was explanatorily remarkably successful:

An until that time unexplainable phenomenon (the increased visual sensitivity of patients suffering from hysteria) became explainable by the N-ray.

The $\mathrm{N}$-ray promised to explain parapsychological phenomena in a physicalist manner (A. d'Arsonval came up with a physicalist account for the aura alleged to surround human body; Kéraval offered an explanation of telepathy by N-rays).

4. Most importantly, the discovery was empirically massively reinforced:

The experiments were successfully replicated (at least 40 researchers demonstrated experimentally the existence of the N-ray between 1903 and 1906).

Several scientists managed to specify, extend and elaborate Blondlot's discovery and new scientific problems were inspired by it (why certain materials radiate and others do not etc.).

The presence of the $\mathrm{N}$-ray was experimentally demonstrated in other fields: first in physiology then in psychology (A. Charpentier found that human nervous system emits such radiation; di Brazza claimed to have discovered rays emitted by the active brain).

d'Arsonval experimentally localised the source of emission in the Broca-centre of the brain.

A quantitative correlation was found between the intensity of the physical-psychical activities and the strength of the radiation emitted by the human body.

By increasing mental or physical activity, physiologists seemed able to manipulate causally the emission and the quantity of the N-ray.

J. Becquerel's control experiments showed that anaesthetisation of the nerves and contraction of the muscles can suppress $\mathrm{N}$-ray emission.
Looking at the list of scientists, institutions, publications, experimental evidence and successful applications, one does not see a solitary scientist's self-delusion. What can be seen is rather a hot research area, a rapidly developing interdisciplinary field that quickly produces experimentally reinforced, quantified results promising solutions to several previously irresolvable problems. As the community constituted by the endorsement of Blondlot's methods consisted of researchers working in north-France (University of Nancy; the Sorbonne, the Institut Général Psychologique; Académie des Sciences in Paris), I am going to refer to it as the N-F community. Let us see how other scientists reacted on the claims this community published.

\section{Failed attempts at an experimental replication}

Several physicists at different laboratories in Germany, England and the USA (the G-A community, for short) made several abortive attempts to replicate the published experiments. The failures were explained in two different ways.

1) Incompatibility with the received knowledge Some accounts referred to the irreconcilability of Blondlot's experimental results with the then known properties of radiations. $\mathrm{H}$. Rubens, for instance, warned that Blondlot's assertion that the $\mathrm{N}$-ray is an electromagnetic radiation of long wavelength that travels through a $0.1 \mathrm{~mm}$ window was incompatible with Maxwell's theory (Nye, 1980: 131). According to G. Sagnac's calculations the wavelength of the $\mathrm{N}$-ray must have been so long that it could not pass through the quartz lens Blondlot used (Nye, 1980: 132). The properties of the N-ray were incompatible with V. Schumann's discovery from which it followed that the air and the quartz lens would completely absorb a radiation with the given properties therefore it could not have been seen without a vacuum spectrograph (which Blondlot did not use). C. C. Schenk (1904) pointed out that if the beams traverse a 5 $\mathrm{mm}$ wide slit then after passing through the prism they must become so broad that their intensity will considerably decrease therefore they cannot be separated from each other. He concluded that "under the conditions of the experiment it would 
hardly be possible to detect the existence of separate beams at all" (Schenk, 1904: 487).

The gist of these objections was that Blondlot's experimental results contradicted the received physical knowledge of the day therefore it could be a priori known that such rays could not exist. This argument, however, was not conclusive for two reasons:

a) As mentioned above, other experimenters reinforced Blondlot's outcomes, hence despite the a priori arguments, there was a posteriori evidence for it.

b) Radiation-physics at that time was a new field thus the so-called "received" knowledge was not confirmed firmly enough to adjudicate Blondlot's outcomes by it. Accordingly, Blondlot was quick to reverse the argument: the incompatibility of his experimental outcomes with the theory suggests that something is wrong with the received knowledge, therefore the strange properties of the $\mathrm{N}$-ray indicate that he had hit upon something important. So when Wood expressed doubts that "a ray bundle $3 \mathrm{~mm}$ in width could be split up into a spectrum with a maxima and minima less than 0.1 mm apart...", he responded that "this was one of the inexplicable and astounding properties of the rays" (Wood, 1904: A 82-86). ${ }^{2}$

\section{2) Concerns about the method}

Several members of the G-A community were tempted to think that the bizarre properties are to be taken as evidence that the experiments had gone wrong. Thus, taking up Schenk's invitation that the scientific community should "... direct attention ... to certain experimental precautions not sufficiently observed ... by Blondlot" (Schenk, 1904: 486) the second group of arguments targeted the experimental method Blondlot applied.

a) O. Lummer gave a lecture in the Deutsche Physikalische Gesellschaft (Nov. 1903) in which he argued that the brightenings the experimenters sensed were due to the different light-sensitivities of the cones and rods of their retina, thus the whole phenomenon was but some illusion (Lummer, 1904a: 280). In the discussion that took place at the 76. Naturforscherversammlung zu Breslau in 1904, he concretised his objection claiming that until the N-ray was objectively justified, he (together with $\mathrm{H}$. Rubens) reserved the right to attribute the phenomena exclusively to physiological and psychological causes (Lummer and Weiss, 1904: 676). Eventually, at the sitting of the Deutsche Physikalische Gesellschaft he explicitly declared that "Blondlot's experiments may be almost exactly imitated in their effects without employing any source of illumination whatever" (Lummer, 1904b: 378, my italics). This meant that Blondlot's experimental method was not objective because he failed to exclude the experimenter's distorting effect from his experiments.

b) A. A. Swinton was not as harsh. He admitted that the brightness of the screen changed, but experimentally demonstrated that this was caused by the heat of the instruments (Nernst lamp, Auer burner) used (Swinton, 1904: 412). Thus, contrary to Lummer, he "proved" that there was an external cause, it only was not the $\mathrm{N}$-ray. This again meant that the experiment was technically sloppy because Blondlot took a noise to be a sign.

c) S. Hooker conducted a control experiment keeping a container filled with hot water close to the screen, but he found that "there was absolutely no brightening" (Hooker, 1904: 686). On the other hand, putting the screen among the branches of a mimosa plant he experienced increased luminosity, thus he concluded that organic beings and what he called "human ray" caused the brightening. With this he not only admitted the presence of an external cause, but came quite close to Charpentier's, di Brazza's, and d'Arsonval's "physiological radiation" that they had identified as N-ray.

In sum, these experiments brought incompatible results that, contrasted with the successful replications of the N-F community, gave the impression that the discussion about the existence of the $\mathrm{N}$-ray have reached a deadlock.

\section{Breaking into the experimenter's regress}

The G-A community did not acquiesce in this thus they opened a new line of attack. They took 
Blondlot's occasional replay to his critics that they fail to see the brightenings because of their "lack of sensitiveness" (Wood, 1904: A 32-33) to mean that his experiments presuppose some extraordinary ability. Swinton noted with a tongue in cheek that "those who have unsuccessfully tried the experiments can only imagine that ... they are only visible to certain individuals" (Swinton, 1904: 412, my italics). Others went sarcastic: "I am at a loss to find any other explanation of M. Blondlot's result than that he has come across a radiation to which some men are blind and others not so" wrote J. B. Burke (Burke, 1904a: 365 - my italics). It was he who in another paper called the N-ray a "mysterious" phenomenon (Burke, 1904b: 198) and repeated this qualification at the $74^{\text {th }}$ meeting of the British Association for the Advancement of Science where he gave an account of his efforts to reveal the nature of this "mysterious radiation" (Discussion, 1905: 468). The efforts mentioned included an investigation in which he "had tried the vision of numerous persons, but in no case was there satisfactory evidence of any external action upon the sight" (Discussion, 1905: 468). This was again a clear allusion to the extraordinary ability that Blondlot was thought to had made a precondition of proving the existence of the N-ray. Eventually, A. Turpain declared boldly that "if the N-rays can only be observed by privileged rarities, then they no longer belong to the domain of experimentation" (quoted by Nye, 1980: 144, my italics). Reading Wood's report about his visit to Blondlot we see that he was not free of this "expectation bias" at all: he went to see the laboratory "in which the apparently peculiar conditions necessary for the manifestation of this most elusive form of radiation appear to exist" (Wood 1904: A 6-8, my italics).

This pushing the N-F community toward irrationalism seemed to allow a spectacular solution to the problem of contradictory experimental results. The instrument Blondlot used came to be conceived as being composed of two parts: one physical (the spectroscope) and one human (Blondlot's mysterious sensitivity). Since the G-A community used a different (exclusively physical) instrument, the data the two communities produced were in fact not incompatible, but simply different. To demonstrate this, in the darkened laboratory Wood allegedly took the prism out of the spectroscope. This trick disabled the physical part of the instrument, leaving just the human one that even according to Blondlot detected only what the physical part projected on the screen. In Wood's report, Blondlot went on reading the spectrum of the refracted $\mathrm{N}$-ray as if nothing had happened. So the fact that he saw the non-existing effects proved that not his visual ability, but another of his faculties, namely his imagination worked there, thus the data he saw were irrelevant.

Mentioning Wood's taking out the prism I used the adverb "allegedly" because the only documentation we have of this action is what $M$. Ashmore called "the tale of the Removal of the Prism" (Ashmore, 1993: 67). As he pointed out, we have to rely exclusively on Wood's testimony concerning when the prism was in or out and how these physical states correlated with what Blondlot said he was seeing. Wood claimed he had taken the prism out of the spectroscope thus no visible spectrum could exist; Blondlot, however, continued to see the spectrum therefore he was sure that the prism must have been in place (cf. Ashmore, 1993: 86). This means that Wood simply replaced the original problem (whether the replicated experiments failed to show N-rays because there is no such radiation, or because Blondlot's experiences were caused by experimental sloppiness) with a new equally undecidable one (how Blondlot's claims were related to the prism's alleged ins and outs).

The situation was what later came to be called the "experimenter's regress" (Collins, 1992: 83-89). The disagreement between the two communities seemed irresolvable by the standard means of science: Blondlot's positive results could be valid detections and other physicists' inability to replicate could be caused by their using an inappropriate (exclusively physical) instrument - or the other way round: their instrument was adequate and they did not see the $\mathrm{N}$-ray because it did not exist. The question at that time was undecidable. All the less, because by its rash publication Wood's act became irreplicable as well, since after what happened, Blondlot (or other N-rayists) could not be expected to report their private sensations of extremely delicate stimuli with the original unsuspecting innocence (cf. Ashmore, 1993: 90). 
The only chance to break into such an experimenter's regress is to find "a criterion ... which is independent of the output of the experiment itself" (Collins, 1992: 83). Wood found such a criterion, namely the authority of the $G-A$ community that approved his "tale of the Removal of the Prism" as a scientific demonstration of the fact that Blondlot's complex equipment was inappropriate. Thereby the G-A community was justified in saying that the $\mathrm{N}$-ray did not exist since the proper instrument could not detect it.

Notice that this breaking into the experimenter's regress was made possible by two factors:

a) The G-A community's endorsement of the "tale of the Removal of the Prism", and

b) the community's interpretation of Blondlot's words on the visual capacity needed for the success of the experiment.

It is obvious that without the G-A community's authoritative support Wood's procedure would never have qualified as a "scientific" proof, let alone an experimentum crucis. He unabashedly presented conditional formulations of unjustified assumptions as incontrovertible evidence: "It appears to me that it is quite possible that..." (Wood, 1904: A 52-56, my emphasis), or "I feel very sure that if a series of experiments were made... the source of error would be found" (Wood, 1904: A 62-67, my emphasis). In vain produced Blondlot photos showing an increased brightness on the unshielded half of photo-sensitive plates exposed to the N-rays, they were brushed off with the remark that the photos "were made, it seems to $m e$, under conditions which admit many sources of error..." (Wood, 1904: A 44-45, my italics). He completed rhetorical manipulation and neglect of facts with wild exaggeration. According to the report, when Blondlot was asked to indicate when he saw changes in the brightness while Wood was alternately blocking the source of the N-ray, "in no case was a correct answer given" (Wood, 1904: A 36-37 - my italics), and Blondlot "was almost 100 per cent wrong" (Wood, 1904: B 14, my emphasis). In a similar test "in no case was a correct answer given..." (Wood, 1904: A 36-37, my italics). Even if we assume that, seeing no real signals, Blondlot gave random answers, Wood still would have owed an explanation why the usual probability distribution of random choices was so extremely distorted. He, however, did not have to care about even the most basic requirements of an empirical refutation. The G-A community backed unanimously his claim that this action was the "crucial and most exciting test" (Wood, 1904: B 25) proving that Blondlot could not see traces of the N-ray.

The same went for the short and quick concluding part of the "falsification process". Having spent hardly more than three hours in Blondlot's laboratory, Wood returned to Paris and next day he sent a report to the Nature. Leaving all the usual precautions aside, this was published immediately. There was no peer review process, the report came out as a "letter to the editor". Despite providing no detailed account of a systematic and repeatable test, the report was immediately given full credence. Indeed, it was republished in leading scientific journals of the field in quick succession (September: Nature, October: Revue Scientifique, December: Physicalische Zeitschrift) making sure that everyone concerned be authoritatively informed about the debunking. The whole action was carried out in a desperate hurry and was in startling contradiction with the advertised "organised scepticism" of science: the meeting in Cambridge at which Wood's visit was decided took place in late August 1904. He visited Blondlot's lab $21^{\text {st }}$ September, and his letter in the Nature came out the $29^{\text {th }}$. The completing phase of the whole $\mathrm{N}$-ray affair was then the consolidation of the contemporaneous interpretation. Historians of science subsumed the case under the category of "pathological science" (Langmuir, 1989).

\section{What was at stake?}

Considering the importance of the question whether there was or there was not a further kind of electromagnetic radiation one finds difficult to comprehend why such a large-scale operation was launched. Not a fundamental theory was refuted, both the existence and the non-existence of the $\mathrm{N}$-ray fitted perfectly into the basic physical views of the era. Thus it is not easy to get rid of the conjecture that the serial of publications in the 
leading physical journals was rather a dramatic demonstration.

To see what happened, recall that Blondlot's opponents spoke about some mysterious ability he allegedly relied on. In fact, however, Blondlot never referred to any extraordinary capacity. What he said was that the stimuli in the $\mathrm{N}$-ray experiment were just above the human perceptual threshold, therefore the observer should look at the screen as "an 'impressionist' painter", and that "to attain this requires some practice, and is not an easy task" (Blondlot, 1905b: 83, my italics). That some sensations require previous practice was not an unheard idea in the psychology of sensation at that time. Blondlot even quoted Helmholtz saying that certain sensations "demand much practice and consequently many facts of this nature cannot even be observed without long preliminary practice ... On many points, therefore, we are restricted to the observations of very few individuals" (Blondlot, 1904: 24211). He echoed this last sentence saying "a few person succeed at once, others after more or less practice ... a few never succeed" (Blondlot, 1904: 24211). It is perfectly clear that his remarks on the personal differences in seeing referred to an acquirable skill and not to some extraordinary gift.

Anyhow, skills were not admitted into the methodology of experimental physics of the time either. The idea that the success of an experiment depends on the active intervention of the experimenter seemed so absurd to the contemporaries that it overshadowed the difference between an inborn ability and a learnable skill. At that time, to use one's personal skill appeared a serious deviation from the norm that the cognitive process should be strictly separated from the subjectivity of the epistemic agent. This was the taken-for-granted conception of the "mechanical objectivity" (Daston and Galison, 2007: 115-190) that dominated the $19^{\text {th }}$ and early $20^{\text {th }}$ century science. The physicists were convinced that the experimenter must act as a "will-less machine", like a camera that was believed "... to offer images uncontaminated by interpretation" (Daston and Galison, 2007: 121, 139).

To put it bluntly, the N-F and G-A communities were committed to different epistemic systems. Their debate was not about a contingent fact (whether there is N-ray or not), but about the legitimate epistemic standards to be used for the justification of scientific claims in experimental physics. They had no conceptual problems, they shared their cognitive aims, values, theoretical backgrounds, and were committed to the general method of experimental justification, to which they deployed standard instruments. They ascribed identical meanings to the crucial terms like 'radiation', 'electromagnetism', 'spectroscope,' 'wavelength', 'prism' etc., still they assigned contrary epistemic values to the claim "the N-ray exists". The standards of objectivity they used differed in at least three interconnected respects:

a) What does the objectivity demand: the mechanical exclusion of the experimenter or rather a participative attitude?

b) Consequently, what is the appropriate attitude of the epistemic agent: should $\mathrm{s} /$ he be passive or interventionist?

c) What is to be regarded a proper experimental instrument: is it exclusively physical or can it be supplemented by human skills?

These standards determine the conditions under which experimentally produced beliefs count as justified, consequently the one concerning the existence of $\mathrm{N}$-ray may take opposite, but equally rationally certified epistemic values. The partisans of the mechanical objectivity found it blatantly obvious that "objective" meant "being determined exclusively by the investigated object" and any interference of the epistemic agent leads to prejudiced, biased, therefore subjective results. Lummer concretised this general conviction when he declared that the existence of the $\mathrm{N}$-ray could only be admitted if it was "incontestably proved by means of objective instruments of precision" (Lummer, 1904b: 380, my emphasis). Blondlot realised that in order to be an objective instrument the spectroscope needs to be completed with a trained eye capable of noticing feeble signs, discern subtle differences, distinguish between sign and noise. Without such a skill, he thought, the spectroscope did not show the fine effects of reality on the screen therefore it was not an "objective instrument of precision". Indeed, because of their taken-for-granted principle of mechanical 
objectivity, Blondlot's opponents were not in the position to be able to debate his claims by empirical arguments. As it expressly forbade them to acquire the necessary skill, they could not be sure that the screen in fact did not brighten or their eyes were simply not sensitive enough to notice its actual brightenings. That is why Wood had to resort to an indirect argument claiming that the "tale of the removal of the prism" proved that his imagination deluded Blondlot, therefore it did not matter what sensations he had.

Briefly, both Blondlot and his opponents could rationally think they proved their case by objective experimental data. Objectivity, however, meant different things for them so they justified their contradicting propositions by applying different standards. That is exactly what we usually call epistemic relativism that, according to Boghossian (2006), is comprised of three interconnected theses:

1) "Epistemic non-absolutism": there are no objective empirical data or logical inferences that unequivocally justify a belief.

2) "Epistemic relationism": the epistemic value is always related to some epistemic system.

3) "Epistemic pluralism": "There are many fundamentally different genuinely alternative epistemic systems, but no facts by virtue of which one of these systems is more correct than any of the others" (Boghossian, 2006: 73).

The G-A community put up a naive defence against relativism attempting to refute the first thesis by presupposing the absolute cognitive power of reason and/or experience. They hoped that, like in he case of semantic relativism, by the observation of the situations in that beliefs arise and by the application of rational assessment they gain uniform justified beliefs, thereby the second thesis becomes invalid, hence the third irrelevant. When such attempts fail, usually there seems to remain no alternative but to admit that "there are no absolute proofs to be had that one scientific theory is superior to another: there are only locally credible reasons" (Bloor, 1999: 102). As an expression of a general theoretical position this assertion may be true. Applied specifically to science, however, the expression "locally credible reasons" calls for a special interpretation.
Was experimental physics at the beginning of the $20^{\text {th }}$ century in fact pursued by communities that could cherish their own "epistemic norms" lending "local credibility" (Seidel, 2014: 143)? Not at all. The contemporary physicists sprang to the defence of the consensual standards of objectivity. The quick and easy approval of Wood's fishy debunking, the replacement of argumentation with rhetoric, the hasty publication of the dubious "refutations", and the neglect of the difference between an inborn gift and an acquired skill demonstrates that epistemic relativism was not considered a viable option. The physicists of the G-A community did not look upon themselves as representatives of one of the possible epistemic systems, but as the guardians of scientificity and rationality as such. This suggests that science makes an exceptional sort of culture in which the inference from non-absolutism to localism is not automatically licensed.

\section{Local communities and disciplinary cultures}

Galison is convinced that the attitude of tolerant discussion and cooperation between incommensurable cultures of science "offers an alternative both to the picture of crazy-quilt fragmentation and to one of homogenous unification" (Galison, 1997a: 51, my italics). Accordingly, as the G-A and N-F communities had "distinct cultures" (Galison, 1997a: 51) with "differences in classification, significance, and standards of demonstration" (Galison, 1999: 146, my italics), their integration would have required some hybrid language facilitating rational "exchanges (coordination), worked out in exquisite local detail, without global agreement" (Galison, 1997a: 46). In fact, however, we find no attempt either at establishing it or at obtaining interpretive expertise. What could have been a better occasion for such a local coordination than the téte-a-téte between Wood and Blondlot? Still, no attempt was made at that. What happened was a rather belligerent intervention and a truculent disqualification of the deviant standards and their advocates. Instead of steering a middle course between fragmentation and unification we see a hard push for the latter. 
Looking for an explanation we find the difference between understanding the meaning of sentences expressing beliefs and justifying the normativeness of epistemic standards. The two groups had no problems with the former, but did not even try to come to terms with the latter. The rational resolution would have taken arguments and reasons, however, the binding force of arguments and reasons would have been provided by the very standards they were expected to be arguments and reasons for. So the usual ingredients (genuinely different epistemic standards + "norm-circularity" (Seidel, 2014: 137-138) of epistemic relativism were given. The impossibility of a metajustification of one of the set of standards prevented a rational debate between the two groups. This, however, did not lead to epistemic relativism. As Kusch puts it, "[a]ll forms of epistemic relativism commit themselves to the view that it is impossible to show in neutral, non-question begging way that one 'epistemic system' (...) is epistemically superior to (all) others." (Kusch, 2017: 4687). These, however, are the philosophical conditions only. Their presence provides only the necessary but not the sufficient conditions of a local relativism.

The epistemic standards are "fundamental commitments which are (...) immune to rational evaluation" (Pritchard, 2016: 66, my italics) hence their endorsement presupposes a culture, tradition, customs or socialisation. This seems to match the main argument for the extension of cultural relativism onto science, namely that "... science is not above culture; it is part of culture" (Livingstone, 2003: 180). This seems to entail relativism since as there are different human cultures there must be scientific traditions as well that differ from each other in their entrenched commitments to various epistemic systems. I argue, however, that science is a special kind of culture that does not tolerate diverse commitments at the same time.

The reason is that contemporary Western science consists of disciplines that have been getting unified since the $17^{\text {th }}$ century on. An integral part of the disciplinary cultures this historical process has brought about is the principle that no synchronous alternative epistemic systems are tolerated. The unity is not established or preserved by reference to neutral experience and universal reason: it is consensual, historically changing and is confined to disciplines. But it creates shared commitments.

The unification I refer to is certainly, not that complete one the neopositivists dreamt about. Their reductionism, verificationism, and methodologism are not part of the project. It is acknowledged that reality may be too complex to approach every region of it by the same methods. Further, it is not imagined that every research groups should take the same particular metaphysical assumptions for granted and it is not hoped that scientific research will eventually produce a grand unified theory of the world (Dupré, 1993; Cartwright, 1999.). The unification I have in mind concerns the epistemic systems (Seidel, 2014; Kusch, 2017) or methodological assumptions (Longino, 2002: 184-189) that select and evaluate evidence and assess scientific statements. This unification is not based on philosophical criteria, it is rather a historical process that brings about "arational hinge commitments" (Pritchard, 2016: 89-103) confined to disciplines. The N-ray case is revealing because it makes visible how, despite lacking some higher-level epistemological principle, the majority of the experimental physics community defends the epistemic unity of their discipline by imposing their uniform standards of objectivity upon a deviant minority.

\section{Spatial - local}

The unification of the disciplines is a historical process entailed by the development of communication that creates place-independent communities. It seems natural to think that local scientific communities come about from the unification of individual efforts. People of the same geographical region who are beset with similar problems compare the ideas and methods they individually hit upon, select the best ones, complete, correct, reinforce or refute each other's views. Thus individual researchers get unified into local epistemic communities kept together by personal relations.

When spatialists describe science they seem to have such communities in mind: "science is always an ancient Chinese, a medieval Islamic, an early modern English, a Renaissance French, a 
Jeffersonian American, an Enlightenment Scottish thing..." (Livingstone, 2003: 13). When, however, they form their general thesis about the localist nature of science they assume, without offering any reason, that the integration process stopped at the level of the local communities. Why would it be so? Certainly, there were contingent practical reasons for the existence of isolated local communities until communication among distant regions was technically difficult and rare. When, however, the facilities of travel, correspondence and publication created dense communicational connections among physically remote communities the process that brought them about simply went on. Urged by curiosity, the spirit of competition, the quest for learning, etc. people doing philosophy of nature in Europe tended to get in touch in an increasing measure from the $17^{\text {th }}$ century on. Correspondence, visits, public experiments, journals, learned societies and academies connected these people irrespective of the geographical place they happened to live in. Invisible colleges emerged that, as described by Price and Crane, are loose, informal assemblages of people, held together by intellectual proximity: regular communication, exchange of preprints, conferences, visits, sameness of the literature read etc.

This spontaneously emerging epistemic homogeneity became institutionalised in the $20^{\text {th }}$ century when a worldwide institutional system (universities, academies, learned societies, peer review system etc.) was developed (Drori et al., 2003; Schofer, 2004). This system takes care of the selection and preservation of the epistemic standards of science by:

a) A standardised knowledge-transmission system that ensures uniform cognitive socialization.

b) An artificial virtual space for constant communication: journals, conferences, workshops etc.

c) A constant migration of persons. Scholarships, visiting scientists, workshops, conferences, summer schools etc. keep up dynamic personal contacts, blend ideas and practices.

d) The strict separation of the local contexts of discovery and the global context of justification ensures that the locally embraced results have to gain accreditation from the whole disciplinary community, therefore justification must observe the actually endorsed non-local criteria.

Thus, despite being unable to offer philosophical arguments for the unity of science as such, we can observe a historical process and an institutional system that bring about the epistemic unity of the researchers dealing with the same problems. Ironically, despite emphasizing that "a whole body of recent empirical and theoretical work" shows "the local, situated and embedded nature of science" (Shapin, 1998: 6), spatialists tend to overlook the historical and institutional homogenising tendencies. The historians of science are certainly justified in their focusing "on the local institutional setting of science ... and on the particularities of the practice that characterise it" (Golinski, 1998: 55). This attitude, however, became obsolete with the emergence of disciplinary communities with which cultural relativism ceased to coincide with spatialism in science. Thanks to the communicational connections, it was no longer important where scientists were geographically located. What made them an epistemic community was that their practices were entrenched in non-local institutions that made them conceptually, methodologically and epistemologically united.

Before outlining how this change effected nongeographical localism in science it will be useful to distinguish between three senses of the adjective "local" in epistemic contexts:

LOC1 The most fundamental sense of localism is non-absolutism: "Rational evaluation is ... an essentially local activity, one that always take place relative to arational hinge commitments" Pritchard 2016, 103). Pritchard calls this the "essential locality of rational evaluation". This locality is strictly epistemic and means that all epistemic evaluation presupposes some epistemic system.

LOC2 Cultural relativism was first motivated by the spatial meaning of localism. Norms are developed by geographically isolated communities hence the beliefs' credibility is provided by "specific local causes" (Barnes and Bloor, 1982: 23).

LOC3 Finally, localism is contrasted with "global relativism" that includes every sphere of 
culture (moral, cognitive, aesthetic etc.). Contrasted with this, local relativism means that one is relativist "with respect to some designated domains" (Krausz, 2011: 74) where more than one genuinely different epistemic system prevail.

With this conceptual articulation in hand we can refine the general statement that the only "characteristics all knowledge systems share is localness" (Watson-Verran and Turnbull, 1995: 116). It is especially important if "localness" is applied to science. Scientific disciplines are obviously local in the first sense: theories and statements cannot be assessed "simpliciter" (McKenna, 2017: 172) only in the context of some epistemic system. As indicated, science ceased to be local in the second, spatialist sense by the emergence of the disciplinary communities. In what follows, I intend to argue that scientific disciplines are not local in the third sense either, i.e. the concretised version of the above statement, namely that the "fundamental characteristic of scientific knowledge is its localness" (Turnbull, 1996: 6) does not apply to the scientific disciplines. My claim is that different epistemic standards may turn up in disciplinary communities, but their existence is transitory only, the synchronous unity of the fundamental commitments is preserved.

\section{The no-tolerance principle in disciplines}

Why I claim that the differences of standards are transitory in disciplines is shown by the $\mathrm{N}$-ray case. At first glance the incompatible experimental outcomes back the localist claim that it is "useful to talk about the difference in cultures between the interacting groups that participate in physics" (Galison, 1997b: 669, my italics). However, the cruelty of the eradication of this discrepancy is a clear sign that the culture of science does not tolerate synchronous subcultures in the same field for long.

Why science is intolerant in this respect is understandable from the fact that its main characteristic since the emergence of disciplinary communities is non-individuality: knowledge is manufactured by a series of interactions like collaboration, critique, adjudication, making use of data produced by others, evaluation of claims to decide about publication, grants, jobs etc. The consequence of the constant interactions is the emergence of a shared stock of ideas, a conventional set of standards, concepts, authorities, common practices, and standard techniques. Certainly, science is a complicated epistemic activity, thus the consensus is never complete, groups may establish local standards, schools and local traditions may come about. Evidence may be insufficient to decide about metaphysical assumptions or about the effectiveness of a methodological innovation. Rival hypotheses and alternative methods may coexist for a while, conceptual, and methodological innovations are suggested etc. However, the necessary diversification of expertise, the effectiveness of the distribution of cognitive labour, the enormous quantities of data, huge instruments, escalating costs of research, and coordinated research programmes of different laboratories make constant communication and interaction inevitable among the scientists pursuing the same discipline.

Division of labour, collaboration and competition are the conditions of producing the best humanly possible knowledge of reality, therefore science cannot consent to incompatible views justified by local commitments in the long run. Disciplinary communities cannot acquiesce in domain relativism because the incompatibility of standards prevents cooperation and competition that are fundamental constituents of this culture and are the preconditions of its cognitive success. The researchers who want to rely on or criticise the theories and data produced by other research teams, or want their own results be used by others, have to adjust themselves to shared non-local standards. That is why one of the principal norms of this culture is to seek homogeneity of fundamental cognitive principles of rationality, justification, and objectivity. Variations are kept under constant pressure and in the long run they are fitted in the general patterns of interaction at any cost: "... one can understand investigative, or scientific, communities as constituted around selections of substantive and methodological assumptions. These selections are a function of both the aims of research and inherited tradition" 
(Longino, 2002: 186, my emphasis). We have seen that when the N-F community did not fit into the inherited tradition of mechanical objectivity it was forced to return to the discipline's "inherited tradition".

As I read it, the N-ray case opens a window on the historical period when the experimental physicists' unified disciplinary community emerged. Referring to the $19^{\text {th }}$ century Britain scene, Livingstone could rightly say that "Bristol science, Manchester science, and Newcastle science are not the same as science in Bristol, science in Manchester, or science in Newcastle. The placename adjectives in these designations attest to scientific practices that were constituted in different ways by different urban cultures" (Livingstone, 2003: 108). By the beginning of the $20^{\text {th }}$ century, however, these cultural differences have disappeared from experimental physics. Physicists working in Baltimore, Breslau, or London discussed the results produced in Nancy as if they had been in the same town. The epistemic homogeneity of the discipline was considered so important that it was defended without seeking a fair mediation between the G-E and the local N-F sub-cultures. No trace of a Galisonian "trading zone", of an "intermediate domain in which procedures could be coordinated locally even where broader meanings clashed" (Galison, 1997a: 46) can be seen. No attempt was made at developing an "interactive expertise" by getting into concrete practical and communicative interactions with the competent local speakers (Collins, 2004). Instead, it was made abundantly clear that the decision about what qualified as "acceptable method," "reliable instrument," "confirming evidence", "proper experimentation", and "criteria of objectivity" was kept under control by the majority of the disciplinary community, and if it intended to be part of this culture, the a local community had to go by them. And it did.

The N-F community undoubtedly regarded itself as part of the same culture of experimental physics as the G-A community. Blondlot and his colleagues never claimed they pursued a different cognitive venture: they regarded their discovery as an addition to the then known kinds of radiation; they published their results in the common forums (journals, conferences) of this culture and their ambition was to have it accepted by the international community. Their results were produced and justified by the standard instruments and in the standard laboratories of the experimental physics, and when Wood asked for Blondlot's collaboration in checking his results, he was ready to receive him right away and agreed to conduct experiments together. After Wood's accusation that they broke the norms of experimentation, the N-F community did not put up a resistance. Not everybody went as far as J. Becquerel who, forgetting about his earlier experiments with the N-ray, suddenly realised that "the purely subjective method employed for testing the effects of the N-ray is antiscientific" (Nye, 1986: 74). Still, the majority tacitly withdrew and prudently changed their field of research. No one replied "we have our own tradition", "we use different standards" or "our claims are just as true as those of the G-A community". They readily admitted that they belonged to the overall culture of science and could not apply local standards.

Naturally, discovery in science is often induced by local factors like authorities, patterns, traditions, co-presence of like-minded people and instruments existing in a local community (Henke and Gieryn, 2008). Thus we can say that "knowledge is constructed in specifically designed and enclosed space" (Golinski, 1998: 98). Cases like the N-ray or cold fusion, however, clearly show that after the emergence of the disciplinary community it is no longer true that "place matters in the way scientific claims come to be regarded as true, in how theories are established and justified" (Livingstone, 2003: 13, my italics). In vain established the N-F community a firm local consensus, in vain were Blondlot's experiments replicated there, this was far from scientific justification. The accreditation process took place in the abstract space created by Nature, Lancet, Scientific American, Revue Scientifique, Physicalische Zeitschrift, and other journals and conferences, and was executed by the broad community of experimental physicists.

There was a clear sign of the disciplinary unity of the experimental physics. Lummer remarked in the Berliner Ophtamologische Gesellschaft's assembly in February 1904 that "up to now only the French researchers have seen those rays" (Lummer, 1904a: 280). A bit later an anonym 
author in the Scientific American put it more sharply: “... why English and German scientists have been uniformly unsuccessful in detecting the strange emanations ... and why French physicists ... furnish more convincing proof of their existence every day"? (Scientific American, 1904: 434). The realisation of the locality of justification could have suggested the presence of "paradigms that pass each other like ships in the night" (Galison, 1997a: 13), however, the thought that a group of physicists had created an autonomous local tradition with particular methods of justification did not even cross anybody's mind. On the contrary, the very fact that somebody was sent to Blondlot's laboratory to check the experiment on the spot, and that Wood expected "peculiar conditions" in the laboratory (Wood, 1904: A 6-8, my italics), made manifest the suspicion that it was not a "placeless place" (Kohler, 2002) as proper science would have required. Surfacing the nonreplicability by standard methods caused a crisis and was considered as "one of those scientific anomalies for which no adequate explanation can ever be offered" (Scientific American, 1904: 434, my emphasis). The understanding of the situation as an unexplainable "anomaly" instead of a difference between epistemically equal, domain specific "local traditions", clearly shows that at the beginning of the $20^{\text {th }}$ century experimental physics was already conceived as a unified discipline with shared fundamental commitments. And the $\mathrm{N}$-ray hypothesis was treated accordingly.

We can theoretically (practically often not) accept that there are multiple ways of living, customs, morals, religious and political views, schools of art, i.e. that broadly dissimilar lay cultures can exist next to each other, without even attempting to reach agreement concerning vital questions. Not so in particular scientific disciplines whose ideal aim is the true or at least the instrumentally most effective description, the deepest possible understanding and the most comprehensive explanation of nature. The only chance to achieve it is interaction that is made impossible by the plurality of epistemic standards. Epistemic relativism is irreconcilable with the collaborative and competitive practice of disciplines therefore the diversity of standards is tolerated only as long as it is inevitable. The members of this culture are socialized to make every effort to unify their epistemic norms to make possible cooperation, division of intellectual labour and critique. The sub-communities that want to have their results accredited have to take part in the selection process that in the long run lets stand only one epistemic system at a time for a disciplinary community. That happened in the N-ray case. Despite their mutual understanding the two communities continued to maintain their contradictory views about the existence of $\mathrm{N}$-ray. As the epistemic standards they used were not rationally discussable, the unification did not happen by argumentation: the majority imposed its standards on the local community by power to restore the unity of the discipline.

\section{Conclusion: the one-dimensionality of scientific relativism}

According to the general definition of epistemic relativism, "knowledge is relative ... because different cultures, societies, epochs, etc. accept different sets of background principles, criteria, and/ or standards of evaluation for knowledge-claims, and there is no neutral way of choosing between these alternative sets of standards" (Siegel, 2011: 201). Like the definitions of relativism in general, this one as well focuses on the impossibility of choosing rationally among the possible alternative epistemic systems. Epistemic relativists regularly assume that since norms and criteria solidify in communal processes that bring about customs and traditions historical and cultural relativism are on a par. The process is the same no matter that the commitments come about by the change of time, physical distance or by the formation of a particular school. Thus historical and cultural relativism is usually regarded different only in their "emphasis on the diachronic rather than the synchronic dimensions of the determinants of thought and action" (Baghramian, 2004: 6, my italics).

My argument was that in science this does not mean that one can pick any of the theoretically possible systems. Historians claiming that the history of science "is on the cusp of a transformation that is about to leave us with a growing number of local historiographies of science" 
(Nappi, 2013: 103) seem right. Indeed, pessimistic induction suggests that the present methodological norms of physics may radically change in the future, hence historical relativism seems a wellfounded phenomenon. However, the acknowledgment of the systematic "... relationships between thought and its social setting" (Ophir and Shapin, 1991: 9) does not entail synchronous relativism in the disciplines of science.

Disciplines does not establish a synchronous unity by the assumed universality of experience (protocol language) or by the inborn general norms of rationality, not even by the G-C-E framework. Here the unity is created by the necessary interactivity of the cognitive process and by the matching "social setting", namely by the institutions of science. Science is a practically and communicationally unified cognitive machinery that brings about synchronously universal epistemic systems in its disciplines. Thus it is not to be denied that social causes play a pivotal role in the assessment of scientific beliefs, and that "true" is replaced by "warranted" or "credible in a community" (Bloor, 1999: 84). But the "causes of belief" that elicit credibility (Bloor, 1999: 84) in science are local only in the sense of epistemic non-absolutism (LOC1), but not in the spatialist (LOC2) or domain-relativist (LOC3) sense.

In other words, relativism remains a legitimate historiographical norm, inevitable if one wants to escape presentism. We should, however realise that the examples spatialists come up with are examples of "historical geography" that do not prove at all that a "geography of contemporary science" should exist. No matter if they work in CERN, in Stanford, or in Tsukuba high energy physicists cooperate, discuss theoretical questions, share experimental data, and exchange experts without difficulty. This suggests that speaking specifically of scientific knowledge we should distinguish two types of relativism.

1) The cognitive culture we call "scientific" is a historical development, the validity of its standards is based on consensus and as such it can change substantially in time. Therefore historical relativism does apply to scientific cognition. The standard examples of the Aristotelian, Newtonian and Einsteinan physics, phlogiston chemistry etc. can be completed by the case of the N-ray: Wood refuted Blondlot's claim by showing that he failed the standards of mechanical objectivity. These standards themselves, however, had to be abandoned soon. In the first decades of the $20^{\text {th }}$ century new instruments were introduced for observing beams, waves, sub-atomic particles, electric and magnetic fields: screens displaying fluorescent lights, cloud chambers showing the visible tracks of electrically charged particles, EEG for recording the electrical activity of the brain etc. These instruments showed flickering lights, shimmerings on screens, photos and charts with very complex and entangled patterns, whose discern and interpretation demanded trained eyes. The epistemically naive principle of "use objective instruments only" or "inborn natural sensational capacities ensure objectivity" did not work any longer. By the middle of the century it has become accepted that experimental practice, observation, language, and calculation alike involve a tacit dimension (Polányi, 1966). Thus a "trained-eye objectivity" came to replace the old mechanical one (Daston and Galison, 2007: 329). ${ }^{3}$

2) Despite being a kind of culture, contemporary science cannot be subsumed under cultural relativism (LOC2 or LOC3). It is exceptional among the cultures, not because of its exceptional methodology or epistemic excellence, simply because it succeeded in establishing universal epistemic systems in several of its disciplines. To put it bluntly, Hopi conception of time, Maori epistemology, African traditional cosmologies, Zande witchcraft and the like cannot be regarded as alternatives to the scientific conceptions. Such examples demonstrate epistemic relativism only if the validity of their separate justificatory frameworks are acknowledged as "scientific". But if they would, then they should not be exempted from the selective pressure prevailing in science and this would result in ceasing the alternative conceptions.

Thanks to the special social setting of the culture of science, all the reasons for relativism revealed by SSK (social legitimation and historical change 
of the norms of rationality; the symmetry thesis; the empirical flexibility of interpretations; social constructivism) can be endorsed without accepting synchronous relativism. This certainly does not mean that such communities obtain an absolute perspective: science remains a human culture, but at least not fragmented into synchronous local points of view, its relativism has only one dimension. 


\section{References}

Ashmore M (1993) The Theatre of the Blind: Starring a Promethean Prankster, a Phoney Phenomenon, a Prism, a Pocket, and a Piece of Wood. Social Studies of Science 23(1): 67-106.

Baghramian M (2004) Relativism. London and New York: Routledge.

Barnes B and Bloor D (1982) Relativism, Rationalism and the Sociology of Knowledge. In: Hollis M and Lukes $\mathrm{S}$ (eds) Rationality and Relativism. Cambridge MA: The MIT Press, pp. 21-47.

Blondlot RM (1904) Explanations and statements concerning N-Rays: A replay to Prof. Wood. Scientific American 151(1): 24211.

Blondlot RM (1905) How the Action of "N" Rays should be observed. In: Blondlot"N" Rays. A Collection of Papers Communicated to the Academy of Sciences (transl. Garcin J), New York and Bombay: Longmans, Green, \& Co, pp. 82-83.

Bloor D (1976) Knowledge and Social Imagery. London: Routledge and Keagan Paul.

Bloor D (1999) Anti-Latour. Studies in the History and Philosophy of Science, 30(1): 81-112.

Boghossian PA (2006) Fear of Knowledge. Against Relativism and Constructivism. Oxford: Clarendon Press.

Burke JB (1904a) The Blondlot n-Rays. Nature 69(1790): 365.

Burke JB (1904b) The Blondlot n-Rays. Nature 70(1809): 198.

Cartwright N (1999) The Dappled World. A Study of the Boundaries of Science. Cambridge: The Cambridge University Press.

Coliva A and Pedersen NJLL (eds) (2017) Epistemic pluralism. Berlin: Palgrave Macmillan.

Collins HM (1992 [1985]) Changing Order: Replication and Induction in Scientific Practice. Chicago: University of Chicago Press.

Collins HM (2004) Interactional expertise as a third kind of knowledge. Phenomenology and the Cognitive Sciences 3(2): 125-143.

Collins HM, Evans R and Gorman M (2007) Trading Zones and Interactional Expertise. In: Collins (ed) Case Studies of Expertise and Experience: special issue of Studies in History and Philosophy of Science 38(4): 657-666.

Collins HM and Evans R (2015) Expertise revisited, Part I. - Interactional expertise. Studies in the History and Philosophy of Science 54: 113-123.

Collins HM and Evans R (2016) Expertise revisited, Part II. - Contributory expertise. Studies in the History and Philosophy of Science 56: 103-110.

Collins H, Evans R and Gorman M (2017) Trading Zones Revisited. arXiv preprint arXiv:1712.06327.

Crespel A and Gélisse Ph (2005) Atlas of Electroencephalography: Awake and Sleep EEG: Activation Procedures and Artifacts. Paris: John Libbey Eurotext.

Daston L and Galison P (2007) Objectivity. New York: Zone Books.

Discussion on N-Rays (1905) Report of the seventy-fourth meeting of the British Association for the advancement of science. London: John Murray, 467-468.

Drori GS, Meyer JW, Ramirez FO and Schofer E (eds) (2003) Science in the Modern World Polity: Institutionalization and Globalization. Stanford: Stanford University Press.

Dupré J (1993) The Disorder of Things. Metaphysical Foundations of the Disunity of Science. Cambridge, MA London: Harvard University Press.

Galison P (1997a) Image and Logic. A material culture of microphysics. Chicago: The University of Chicago Press. 
Galison P (1997b) Material Culture, Theoretical Culture and Delocalization. In: Krige J and Pestre D (eds) Science in the Twentieth Century. Amsterdam: Harwood, pp. 669-682.

Galison P (1999) Trading Zone: Coordinating Action and Belief. In: Biagioli M (ed) The Science Studies Reader. New York: Routledge, pp. 137-160.

Galison P (2010) Trading with the Enemy. In: Gorman M E (ed) Trading Zones and Interactional Expertise. Cambridge, MA:The MIT Press, pp. 25-52.

Golinski J (1998) Making Natural Knowledge. Constructivism and the History of Science. Cambridge: Cambridge University Press.

Gorman ME (2002) Levels of Expertise and Trading Zones: A Framework for Multidisciplinary Collaboration. Social Studies of Science 32(5/6): 933-938.

Henke CR and Gieryn TF (2008) Sites of Scientific Practice: The Enduring Importance of Place. In: Hackett EJ, Amsterdamska O, Lynch M and Wajchman J (eds) The Handbook of Science and Technology Studies. Cambridge, MA - London: The MIT Press, pp. 353 - 376.

Hooker SJ (1904) Human and plant rays. The Lancet. 1904 March: 686.

Klotz IM (1980) The N-ray Affair. Scientific American 242 May: 168-175.

Klotz IM (1986) Great Discoveries Not Mentioned in Textbooks: N Rays. In: Klotz IM Diamond Dealers and Feather Merchants. Tales from the Sciences. New York: Springer.

Knorr-Cetina KD (1981) The Manufacture of Knowledge. An Essay on the Constructivist and Contextual Nature of Science. Oxford: Pergamon Press.

Kohler RE (2002) Place and Practice in Field Biology. History of Science 40(2): 189-210.

Krausz M (2011) Varieties of Relativism and the Reach of Reasons in Companion In: Hales SD (ed) $A$ Companion to Relativism, Wiley - Blackwell, pp. 70-84.

Kusch M (2017) Epistemic relativism, scepticism, pluralism. Synthese 194: 4687-4703

Langmuir I (1989) Pathological Science. Physics Today 42(10): 36-48.

Livingstone DN (2003) Putting Science in its Place: Geographies of Scientific Knowledge. Chicago: The University of Chicago Press.

Longino HE (2002) The Fate of Knowledge. Princeton and Oxford: Princeton University Press.

Lummer O (1904a) Berliner Ophtamologische Gesellschaft. Sitzung vom 18. Februar 1904. Zeitschrift für Augenheilkunde 11: 279-280. Available at: www.karger.de/Article/Pdf/290155 (accessed 22 July 2014).

Lummer O (1904b) Blondlot's n-Ray Experiments. Nature 69(1790): 378-380.

Lummer O and Weiss P (eds) (1904) N-Srahlen. Physikalische Zeitschrift 5(21): 674-677.

McKenna R (2017) Pluralism about knowledge. In: Coliva A and Pedersen NJLL (eds) (2017) Epistemic pluralism. Berlin: Palgrave Macmillan, pp. 171-199.

Nappi C (2013) The Global and Beyond. Adventures in the Local Historiographies of Science. Isis 104(1): 102-110.

Nye MJ (1980) N-Rays: An Episode in the History and Psychology of Science. Historical Studies in the Physical Sciences 11(1): 125-156.

Nye MJ (1986) Science in the Provinces: Scientific Communities and Provincial Leadership in France, 1860-1930. Berkeley, Los Angeles and London: University of California Press.

Ophir A and Shapin S (1991) The Place of Knowledge: A Methodological Survey. Science in Context 4(1): 3-21.

Polanyi M (1966) The Tacit Dimension. Chicago: The Chicago University Press.

Powell RC (2007) Geographies of science: histories, localities, practices, futures. Progress in Human Geography 31(3): 309-329. 
Pritchard D (2016) Epistemic Angst. Radical skepticism and the groundlessness of our believing. Princeton: Princeton University Press.

Schenk CC (1904) Blondlot's the N-rays. Nature 69(1795): 486-487.

Schofer E (2004) Cross-National Differences in the Expansion of Science 1970-1990. Social Forces 83(1): $215-$ 248.

Scientific American (1904) The N-Rays: Are they real or illusory? Scientific American, June 04, 434.

Seabrook W (1941) Doctor Wood: A Modern Wizard of the Laboratory. New York: Harcourt, Brace \& C.

Seidel M (2014) Epistemic relativism. A constructive critique. Palgrave and Macmillan.

Shapin S (1998) Placing the View from Nowhere: Historical and Sociological Problems in the Location of Science. Transactions of the Institute of British Geographers 23(1): 5-12.

Siegel H (2011) Epistemological Relativism: Arguments Pro and Con. In: Hales SD (ed) A Companion to Relativism. Chicester, Malden Ma:Wiley-Blackwell, pp. 201-218.

Swinton CAA (1904) The Blondlot N-rays. Nature 69(1792): 412.

Turnbull D (1996) Cartography and Science in Early Modern Europe: Mapping the Construction of Knowledge Spaces. Imago Mundi 48(1): 5-24.

Turnbull D (2002) Travelling knowledge: narratives, assemblage and encounters. In: Bourguet MN, Licoppe $\mathrm{C}$ and Sibum $\mathrm{HO}$ (eds.) Instruments, Travel and Science. Itineraries of precision from the seventeenth to the twentieth century. London and New York: Routledge, 273-294.

Watson-Verran H and Turnbull D (1995) Science and Other Indigenous Knowledge Systems. In: Jasanoff S, Markle GE, Petersen JC and Pinch T (eds) Handbook of Science and Technology Studies. Thousand Oaks, London, New Delhi: Sage Publications, pp. 115 - 139.

Williams M (2007) Why (Wittgensteinian) Contextualism is not Relativism, Episteme 4(1): 93-114.

Withers C and DN Livingstone (2011) Thinking Geographically about Nineteenth-Century Science. In: Livingstone DN and Withers C (eds) Geographies of Nineteenth-Century Science. Chicago: The University of Chicago Press, pp. 1-20.

Wood RW (1904) The N-rays. Nature 70(1822) (29 Sept.): 530-531.

\section{Notes}

1 The following summary is based on Nye 1980 and 1986, and Klotz 1980 and 1986.

2 I cite the two versions of Wood's paper from the appendix attached to Ashmore 1993 because there the lines are numbered. The text denoted by ' $A$ ' was originally published in Nature 1904; the one marked by ' $B$ ' in Seabrook 1941. The numbers indicate the lines of the respective text-versions published by Ashmore.

3 As an illustration of the change, have a glimpse at the description how the neurologists learn to see an EEG-chart:

"stage I: nothing makes any sense, stage II: you think you understand but you see abnormalities everywhere, stage III: you gain more hindsight. You recognize a spike but wonder if it is actually significant, stage IV: you are finally able to form your opinion, even if it is different from your teacher's. This last stage is the sign you have matured. You have acquired enough experience to have your own opinion and to discuss an EEG (Crespel and Gélisse, 2005: 13)". 